Article

\title{
Binding between Saikosaponin C and Human Serum Albumin by Fluorescence Spectroscopy and Molecular Docking
}

\author{
Yi-Cun Chen ${ }^{1,2,+}$, Hong-Mei Wang ${ }^{1,+}$, Qing-Xia Niu ${ }^{1, *}$, Dan-Yan Ye ${ }^{2}$ and Guo-Wu Liang ${ }^{1}$ \\ 1 Key Immunopharmacology Laboratory of Guangdong Province, Department of Pathophysiology, \\ Institute of Inflammation and Immune Diseases, Shantou University Medical College, Guangdong 515041, \\ China; chenyicun@yeah.net (Y.-C.C.); junebulu@163.com (H.-M.W.); gwliangizzy@163.com (G.-W.L.) \\ 2 Department of Pharmacology, Traditional Chinese Medicine Laboratory, \\ Shantou University Medical College, Guangdong 515041, China; 15917907394@163.com \\ * Correspondance: nqx_1961@163.com; Tel.: +86-754-8890-0380; Fax: +86-754-8890-0192 \\ + These authors contributed equally to this work.
}

Academic Editor: Derek J. McPhee

Received: 11 November 2015 ; Accepted: 21 January 2016 ; Published: 28 January 2016

\begin{abstract}
Saikosaponin C (SSC) is one of the major active constituents of dried Radix bupleuri root (Chaihu in Chinese) that has been widely used in China to treat a variety of conditions, such as liver disease, for many centuries. The binding of SSC to human serum albumin (HSA) was explored by fluorescence, circular dichroism (CD), UV-vis spectrophotometry, and molecular docking to understand both the pharmacology and the basis of the clinical use of SSC/Chaihu. SSC produced a concentration-dependent quenching effect on the intrinsic fluorescence of HSA, accompanied by a blue shift in the fluorescence spectra. The Stern-Volmer equation showed that this quenching was dominated by static quenching. The binding constant of SSC with HSA was $3.72 \times 10^{3}$ and $2.99 \times 10^{3} \mathrm{~L} \cdot \mathrm{mol}^{-1}$ at $26^{\circ} \mathrm{C}$ and $36^{\circ} \mathrm{C}$, respectively, with a single binding site on each SSC and HSA molecule. Site competitive experiments demonstrated that SSC bound to site I (subdomain IIA) and site II (subdomain IIIA) in HSA. Analysis of thermodynamic parameters indicated that hydrogen bonding and van der Waals forces were mostly responsible for SSC-HSA association. The energy transfer efficiency and binding distance between SSC and HSA was calculated to be $0.23 \mathrm{~J}$ and $2.61 \mathrm{~nm}$ at $26^{\circ} \mathrm{C}$, respectively. Synchronous fluorescence and CD measurements indicated that SSC affected HSA conformation in the SSC-HSA complex. Molecular docking supported the experimental findings in conformational changes, binding sites and binding forces, and revealed binding of SSC at the interface between subdomains IIA-IIB.
\end{abstract}

Keywords: saikosaponin C; human serum albumin; static quenching; Radix bupleuri; molecular docking; Chaihu

\section{Introduction}

Serum albumin is the most abundant plasma protein, being present in plasma at a concentration of approximately $40 \mathrm{mg} \cdot \mathrm{mL}^{-1}(\sim 0.6 \mathrm{mM})$ [1]. In addition to blood, about $60 \%$ of the total albumin is also found in tissues and secretions [2]. As a carrier protein, HSA plays an essential role in the binding of drugs, as well as endogenous and exogenous substances, such as fatty acids, bilirubin, and metal ions, in the bloodstream [3,4]. The organic small molecules and proteins interact with each other by means of basic amino acid residues on the protein, including arginine, lysine and histidine residues, and $\mathrm{N}$-terminal amino groups [5]. The interaction between drugs and HSA directly affects the absorption, distribution, free drug concentration, and therapeutic effects [6]. Therefore, drug-HSA 
coupling has been widely investigated in the life sciences, chemistry, pharmacology and clinical medicine [7-10]. In practice, it is very difficult to directly measure drug-protein binding in vivo. In recent years, the binding properties of HSA with drugs have extensively been characterized in vitro by fluorescence spectroscopy.

Chaihu, derived from Bupleurum chinense DC or Bupleurum scorzoneraefolium Willd, has been widely administered for many centuries as a well-known medicinal herb in China, Japan and other Asian countries [11]. The contemporary clinical applications for Chaihu include a variety of diseases, especially liver diseases, for instance, jaundice, hepatitis and liver cirrhosis [12-14]. Saikosaponin C (SSC, CAS Registery Number: 20736-08-7, Figure 1), a triterpenoid saponin, is one of the major active ingredients in Chaihu. Recently, it has been recently reported to exert various biological effects. SSC efficiently inhibits hepatitis and apoptosis, and facilitates the growth, migration and angiogenesis of endothelial cells $[12,15,16]$. Although SSC is one of the major components in Chaihu, and possesses important biological functions, the interaction between SSC and HSA is still unknown.

In the present study, SSC purified from Bupleurum chinense DC was employed as a quencher for HSA fluorescence. Fluorescence, circular dichroism (CD) spectroscopy, site marker competitive experiments, UV absorption, theoretical analysis, and molecular docking studies were performed for the first time in an attempt to investigate the interaction and mechanisms of SSC with HSA. This study should provide useful information about the pharmacology and role of SSC/Chaihu in clinical medicine.

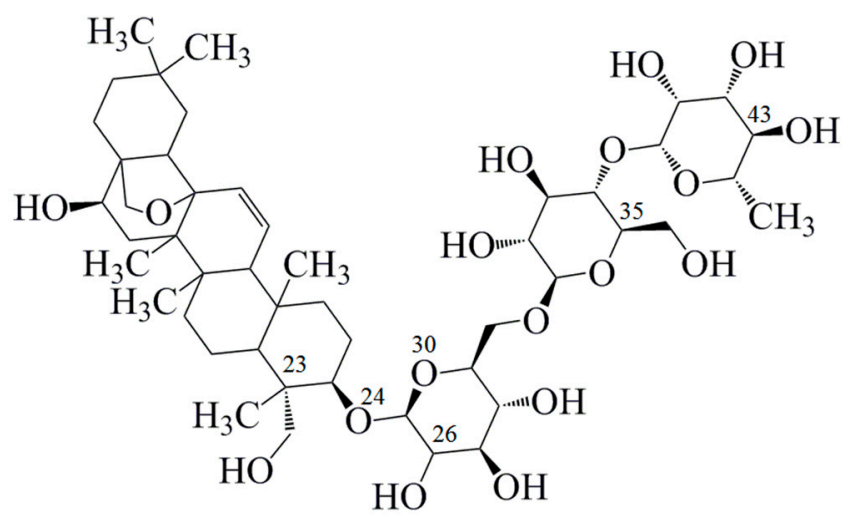

Figure 1. Chemical structure of saikosaponin $\mathrm{C}\left(\mathrm{C}_{48} \mathrm{H}_{72} \mathrm{O}_{17}\right.$, molecular weight 920, numbered using ChemDraw software).

\section{Results and Discussion}

\subsection{Fluorescence Quenching of HSA by SSC}

HSA contains an endogenous fluorophore. At a concentration of $2 \mu \mathrm{M}, \mathrm{HSA}$ showed a strong fluorescence at $26{ }^{\circ} \mathrm{C}$, whereas SSC or the PBS control showed little (Figure 2). Little information is available on the interaction between saikosaponins and proteins. At an excitation of $280 \mathrm{~nm}$, the maximum emission wavelength of HSA was $334 \mathrm{~nm}$, which is in line with previous reports on the binding of other drugs to HSA [3]. Following SSC addition, the fluorescence intensity of HSA decreased regularly with the increase of SSC concentration. SSC did not affect the basic peak appearance of HSA. Importantly, SSC evoked a blue shift of the fluorescence peaks. At $15 \mu \mathrm{M}$ SSC, the shift reached $16 \mathrm{~nm}$, ranging from 334 to $318 \mathrm{~nm}$, suggesting that SSC and HSA bind to each other to form an SSC-HSA complex. A blue shift of maximum emission wavelength reveals an increase of hydrophobic amino acid residues in the microenvironment of the fluorophores, and a red shift indicates an increase of polar residues $[17,18]$. Accordingly, the results as shown in Figure 2 imply that more hydrophobic amino acid groups are formed by the interaction between SSC and HSA. 


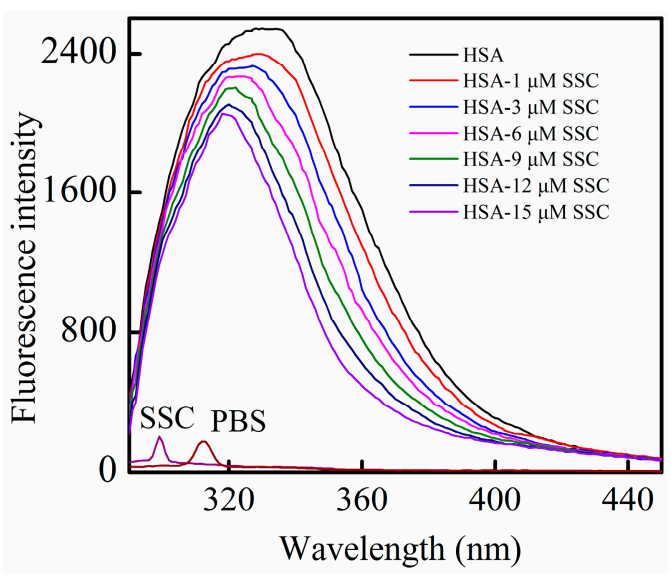

Figure 2. Fluorescence quenching of HSA in the presence of SSC. The observations were performed at $26^{\circ} \mathrm{C} . \lambda_{\mathrm{ex}}=280 \mathrm{~nm} ; \mathrm{C}_{\mathrm{HSA}}=2 \mu \mathrm{M} ; \mathrm{SSC}$, saikosaponin $\mathrm{C}$.

\subsection{SSC-Induced Static Quenching}

Fluorescence quenching is classified into dynamic quenching and static quenching. Dynamic quenching is brought about by intermolecular collision between a quencher and fluorescent molecule at an excited state, in which effective collision and the quenching constant should increase with a rise in temperature. Dynamic quenching does not affect the structure and bioactivity of protein. Static quenching is derived from the intramolecular interaction of quenchers with fluorescent molecules at a ground state, which forms a new composite. In static quenching, the steadiness of new composites decreases with the rise in temperature, since high temperatures cause molecular diffusion and the dissociation of weakly bound complexes. Accordingly, dynamic quenching and static quenching can be distinguished by the response to temperature change. To examine the mechanism of SSC-induced quenching, the Stern-Volmer equation for dynamic quenching was first employed as follows $[19,20]$ :

$$
\frac{F_{0}}{F}=1+K_{q} \tau_{0}[Q]=1+K_{s v}[Q]
$$

where $F_{0}$ and $F$ stand for the fluorescence intensities in the absence and presence of the quencher, respectively. The average lifetime of fluorescent molecules without quenchers, $\tau_{0}$, is taken as $10^{-8} \mathrm{~s}$ [21]. $K_{s v}$, known as the Stern-Volmer quenching constant, is the ratio of the bimolecular quenching rate constant to the decay rate constant of a single molecule $\left(K_{s v}=K_{q} \tau_{0}\right)$. [Q] is the quencher concentration. $\mathrm{Kq}$ is the quenching rate constant of $2.0 \times 10^{10} \mathrm{~L} \cdot \mathrm{mol}^{-1} \cdot \mathrm{S}^{-1}$, which is the limiting diffusion rate constant for the quencher and biological molecule complex [7]. The constant at both $26^{\circ} \mathrm{C}$ and $36^{\circ} \mathrm{C}$ was much higher than the criterion of $2.0 \times 10^{10} \mathrm{~L} \cdot \mathrm{mol}^{-1} \cdot \mathrm{S}^{-1}$, indicating that static quenching is mainly responsible for SSC-induced fluorescence quenching of HSA (Table 1).

Table 1. Quenching reactive constants of HSA by SSC.

\begin{tabular}{ccccc}
\hline Fluorescence & $\mathbf{T}\left({ }^{\circ} \mathbf{C}\right)$ & Detection & $K \boldsymbol{q}\left(\mathbf{L} \cdot \mathbf{m o l}^{-1} \cdot \mathbf{S}^{-1}\right)$ & $\mathbf{R}$ \\
\hline Conventional & 26 & $\lambda_{\mathrm{ex}}=280 \mathrm{~nm}$ & $4.19 \times 10^{12}$ & 0.996 \\
& 36 & $\lambda_{\mathrm{ex}}=280 \mathrm{~nm}$ & $1.17 \times 10^{12 *}$ & 0.965 \\
Synchronous & 26 & $\Delta \lambda=15 \mathrm{~nm}$ & $2.97 \times 10^{11}$ & 0.963 \\
& 26 & $\Delta \lambda=60 \mathrm{~nm}$ & $2.89 \times 10^{12}$ & 0.995 \\
\hline
\end{tabular}

$\overline{\mathrm{R}}$, linear correlation coefficient; ${ }^{*} p<0.05$ compared with conventional fluorescence at $26^{\circ} \mathrm{C}$ by the paired $t$-test.

Moreover, as calculated from Equation (1), the $K q$ for SSC at $26{ }^{\circ} \mathrm{C}$ was much above the $K q$ at $36{ }^{\circ} \mathrm{C}$, confirming that static quenching contributes to SSC-induced quenching of HSA. A plot (Figure 3), 
according to the findings in Figure 2, the Stern-Volmer equation and Table 1 shows good linearity and correlation coefficients, which supports static quenching created by SSC.
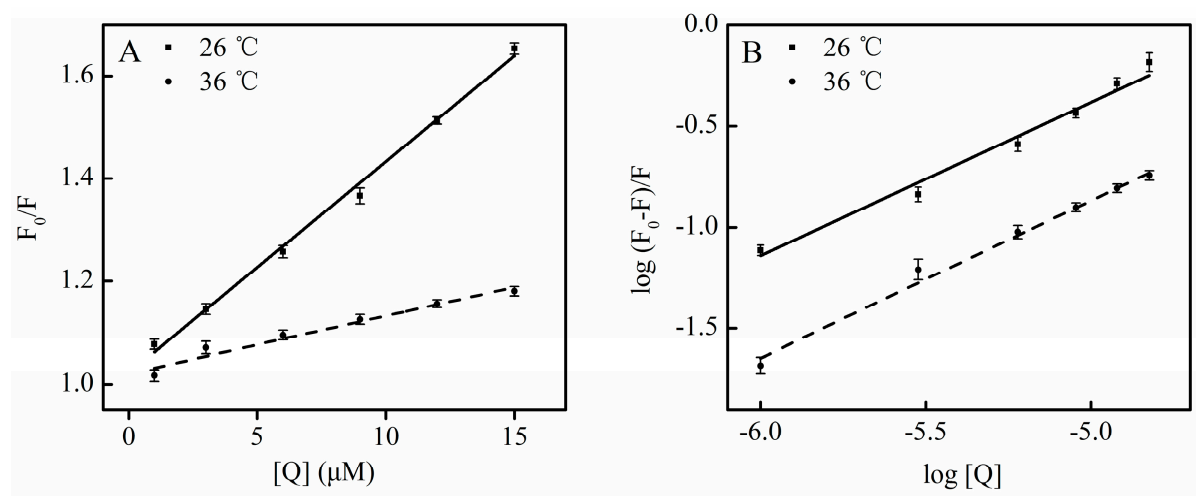

Figure 3. Plots for the interaction of SSC with HSA. Experimental conditions were as described in Figure 2. (A) Stern-Volmer plots for the binding of SSC to HSA; (B) Double-reciprocal plots for log $\left(\mathrm{F}_{0}-\mathrm{F}\right) / \mathrm{F}$ vs. $\log [\mathrm{Q}] ; \mathrm{F}_{0}$, HSA fluorescence intensities without the quencher; $\mathrm{F}, \mathrm{HSA}$ fluorescence intensities with the quencher. [Q], SSC at $0,1,3,6,9,12$ and $15 \mu \mathrm{M}$, respectively.

\subsection{SSC-Induced Conformational Change in HSA}

\subsubsection{Investigation of Synchronous Fluorescence}

HSA fluorescence mainly comes from tyrosine or tryptophan residues. $\Delta \lambda=15 \mathrm{~nm}$ or $\Delta \lambda=60 \mathrm{~nm}$ is characteristic of fluorescence spectra by tyrosine or tryptophan residues, respectively. To clarify the mechanism of SSC-induced quenching of HSA, synchronous fluorescence spectrophotometry was used to obtain fluorescence spectra for tyrosine or tryptophan residues in HSA. At $\Delta \lambda=15 \mathrm{~nm}$, the fluorescence intensity decreased slightly, and a blue shift of about $2 \mathrm{~nm}$ was observed at maximum emission wavelength when SSC concentrations increased (Figure 4A). A blue shift of the ascending branch of fluorescence spectra could be observed, and a blue shift of $4 \mathrm{~nm}$, from 272 to $268 \mathrm{~nm}$, occurred at a maximum point of shift (Figure 4A). These results revealed that SSC induces a conformational change in HSA. As shown in Figure 4B, the peak fluorescence at $\Delta \lambda=60$ nm declined with SSC concentration. Also, a blue minor shift of $1 \mathrm{~nm}$ (from 279 to $278 \mathrm{~nm}$ ) was produced at the maximum emission. These observations revealed that both tyrosine and tryptophan are likely involved in the binding reaction between SSC and HSA. The microenvironmental polarity around amino acid residues can be judged by the shift in the fluorescence spectra of amino acid residues closely related to the protein conformational changes [18]. The blue shift demonstrates that the related residues within HSA are closer, and the range of extension of the peptide chain becomes reduced. Subsequently, the microenvironmental polarity surrounding the residue is converted from hydrophilicity to hydrophobicity, folding the peptide chain and destroying its ordered structure in order to form a new conformation [22]. The changes of spectra for tyrosine or tryptophan were consistent with fluorescence spectra for HSA (Figure 2). Similar to HSA, the Kq at both $\Delta \lambda=15 \mathrm{~nm}$ and $\Delta \lambda=60 \mathrm{~nm}$ was also over the criterion of $2.0 \times 10^{10} \mathrm{~L} \cdot \mathrm{mol}^{-1} \cdot \mathrm{S}^{-1}$, suggesting that both tyrosine and tryptophan residues are most likely associated with static quenching. Additionally, a strong correlation for $\Delta \lambda=15 \mathrm{~nm}$ or $\Delta \lambda=60 \mathrm{~nm}$ was observed in Table 1, which supports SSC-mediated static quenching.

The minor blue shift is likely due to the fact that no direct interaction occurs between SSC and fluorophore in HSA. That is, tyrosine or tryptophan residues are indirectly micro-perturbed when SSC directly binds to the other amino acid residues surrounding SSC, leading to HSA conformation alterations. In order to investigate if there is a direct interaction between SSC and tyrosine or tryptophan residues, the corresponding synchronous fluorescence measurements were performed as described in Section 3.3.3. The results showed that there was no difference, on synchronous fluorescence spectra, 
between tyrosine or tryptophan alone and an SSC complex with each residue (data not shown), suggesting that the direct binding of SSC with the two residues did not exist.
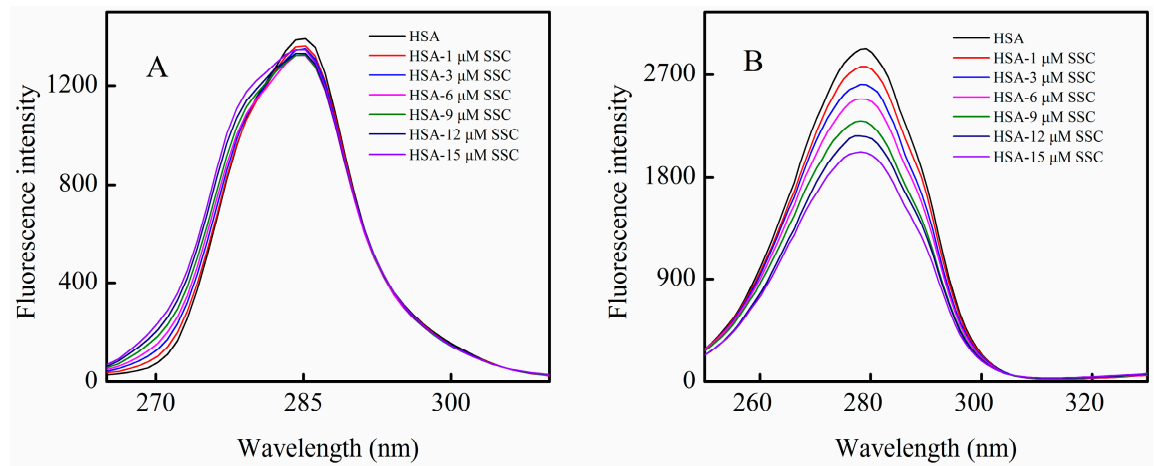

Figure 4. Synchronous fluorescence spectra of the SSC-HSA system. The experimental conditions were as described in Figure 2. A, $\Delta \lambda=15 \mathrm{~nm}$ for tyrosine; $\mathrm{B}, \Delta \lambda=60 \mathrm{~nm}$ for tryptophan. $\mathrm{C}_{\mathrm{HSA}}=2 \mu \mathrm{M}$.

\subsubsection{HSA Conformational Change by CD Measurements}

CD was applied to clarify the conformational effect of SSC on HSA. The $\alpha$-helical content in free HSA and the HSA-SSC complex can be calculated by the following equations [23]:

$$
\begin{gathered}
M R E=\frac{C D(m \mathrm{deg})}{10 C_{p} n l} \\
\alpha-\operatorname{helix}(\%)=\frac{-(M R E)_{209}-4000 C D(m \mathrm{deg})}{33000-4000} \times 100
\end{gathered}
$$

where mean residue ellipticity $(\mathrm{MRE})_{209}, C_{p}, n, 33,000$, and $l$ is the mean residue ellipticity observed at $209 \mathrm{~nm}$, HSA concentrations, the number of amino acid residues, the MRE of a pure $\alpha$-helix at $209 \mathrm{~nm}$, and the path length, respectively. The 4000 is the MRE of the $\beta$-form and random coil conformation cross at $209 \mathrm{~nm}$. Figure 5 shows the negative absorption at near $209 \mathrm{~nm}$ and $220 \mathrm{~nm}$, the $\alpha$-helical characters of proteins, which contributes to $\pi-\pi^{*}$ and $n-\pi^{*}$ electron transfer for the peptide bond of $\alpha$-helix, respectively. The negative peak at $209 \mathrm{~nm}$ slightly shifted to the left. No shift of the peak was observed at $209 \mathrm{~nm}$. The content of $\alpha$-helix and $\beta$-turn increased by $4.9 \%$ (from $55.9 \%$ to $60.8 \%$ ), and $4.8 \%$ (from $5.7 \%$ to $10.5 \%$ ), and $\beta$-sheet and random coil decreased $2.3 \%$ (from $19.1 \%$ to $16.8 \%$ ), and $3.2 \%$ (from $16.0 \%$ to $12.8 \%$ ), respectively. In accordance with the blue shift by fluorescence measurements, the changes indicate that the extension of the peptide chain is partly reduced following the binding of SSC to HSA. This is perhaps due to the fact that SSC perturbs $\pi-\pi^{*}$ and $n-\pi^{*}$ responses, and provokes the rearrangement of the polypeptide network.

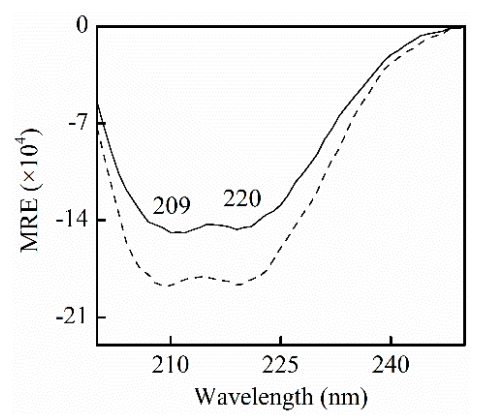

Figure 5. CD spectra for HSA in the absence and presence of SSC in pH7.4, 0.5 M Tris-Cl buffer. $\mathrm{C}_{\mathrm{HSA}}=0.42 \mu \mathrm{M} ; \mathrm{C}_{\mathrm{SSC}}=0.42 \mu \mathrm{M}$. A solid line stands for free HSA. A dotted line is for HSA-SSC. MRE, mean residue ellipticity. 
Few observations on the binding of SSC to HSA are available. $\alpha$-helices and $\beta$-sheets in Figure 5 are consistent with CD spectroscopic studies for free HSA [24]. $\beta$-turns and random coils differed little from some reports [24,25], and could be accounted for by minor differences in experimental conditions. As described by our synchronous fluorescence study, the results indicate that SSC can interact with HSA, and influence its secondary structure.

\subsection{Binding Constants, Sites and Forces between SSC and HSA}

\subsubsection{Binding Constants and Numbers of Binding Sites}

The $K_{a}$ for high affinity binding is higher than $10^{4} \mathrm{~L} \cdot \mathrm{mol}^{-1}$ in order of magnitude. The $K_{a}$ for low affinity binding is lower than $10^{3} \mathrm{~L} \cdot \mathrm{mol}^{-1}$ in order of magnitude [24]. The $K_{a}$ is obtained based on Equation (4) [19]:

$$
\log \frac{\left(F_{0}-F\right)}{F}=\log K_{a}+n \log [Q]
$$

The resulting $K_{a}$ value was $3.72 \times 10^{3}$ and $2.99 \times 10^{3} \mathrm{~L} \cdot \mathrm{mol}^{-1}$. The number of binding sites was 0.79 and 0.83 at $26{ }^{\circ} \mathrm{C}$ and at $36{ }^{\circ} \mathrm{C}$, respectively (Table 2). These indicate that SSC binds to HSA with low affinity, and the binding is weak and easily reversed. No statistical difference in the $K_{a}$ value was observed between $26^{\circ} \mathrm{C}$ and $36^{\circ} \mathrm{C}$, suggesting that the quenching efficiency of SSC to HSA was constant at the tested temperatures. These reveal that SSC can be bound, transported, and released by HSA into target tissues.

Table 2. Binding reactive parameters of HSA by SSC.

\begin{tabular}{lccccc}
\hline Fluorescence & $\mathbf{T}\left({ }^{\circ} \mathbf{C}\right)$ & Detection & $\boldsymbol{K}_{a}\left(\mathbf{L} \cdot \mathbf{m o l}^{-1}\right)$ & $\mathbf{R}$ & $\boldsymbol{n}$ \\
\hline Conventional & 26 & $\lambda_{\text {ex }}=280 \mathrm{~nm}$ & $3.72 \times 10^{3}$ & 0.983 & 0.79 \\
& 36 & $\lambda_{\mathrm{ex}}=280 \mathrm{~nm}$ & $2.99 \times 10^{3}$ & 0.959 & 0.83 \\
Synchronous & 26 & $\Delta \lambda=15 \mathrm{~nm}$ & $0.90 \times 10^{2}$ & 0.986 & 0.57 \\
& 26 & $\Delta \lambda=60 \mathrm{~nm}$ & $1.85 \times 10^{3}$ & 0.989 & 0.77 \\
\hline
\end{tabular}

$K_{a}$, binding constant; R, linear correlation coefficient; $n$, the number of binding sites.

\subsubsection{Identification of Warfarin and Ibuprofen Binding Sites}

Crystallographic analyses show that HSA, a 585 amino acid residue monomer protein, contains three homologous $\alpha$-helical domains (domains I-III), each of which includes subdomain A and B, to comprise six subdomains, IA, IB, IIA, IIB, IIIA, and IIIB. The two subdomains in each domain constitute a hydrophobic cavity [25]. HSA has a limited number of binding sites for a variety of ligands that are typically bound reversibly [1]. Crystal structure analyses also indicate that the two principal regions of ligand binding sites in albumin, site I and site II, are positioned in hydrophobic cavities. Site I, located within subdomain IIA, preferentially integrates with bulky heterocyclic ligands, such as warfarin and, bilirubin $[9,26]$. Site II, located within subdomain IIIA, preferentially recognizes aromatic compounds, and is more active in the accommodation of ligands, for example, digitoxin, ibuprofen, and tryptophan [27].

In order to identify SSC binding sites on the region of HSA molecule, site competitive binding experiments have been commonly carried out. Warfarin (an anticoagulant drug, a marker of site I) and ibuprofen (a nonsteroidal anti-inflammatory agent, a marker of site II) were utilized in the present experiment. Following the addition of SSC into the HSA-warfarin solution, the fluorescence intensity of HSA became significantly reduced (Figure 6A). A shift in fluorescence spectra at the maximum emission wavelength was not found in the warfarin mixture system, while the minor blue shift was observed at $\Delta \lambda=15 \mathrm{~nm}$ or $\Delta \lambda=60 \mathrm{~nm}$ in Figure 4 , suggesting that the binding of warfarin to HSA inhibits the formation of hydrophobic structures surrounding tyrosine or tryptophan residues in HSA-SSC complex. As shown in Figure 6B, following the addition of SSC into the HSA-ibuprofen 
solution, HSA fluorescence intensity weakened dose-dependently. In addition, ibuprofen induced a blue shit of $11 \mathrm{~nm}$ (from $336 \mathrm{~nm}$ to $325 \mathrm{~nm}$ ) in the ibuprofen mixture system, indicating that the hydrophobicity of amino acid residues arises around ibuprofen. The competitive effect of ibuprofen on HSA binding reveals that SSC mainly binds site II (subdomain IIIA) in HSA.
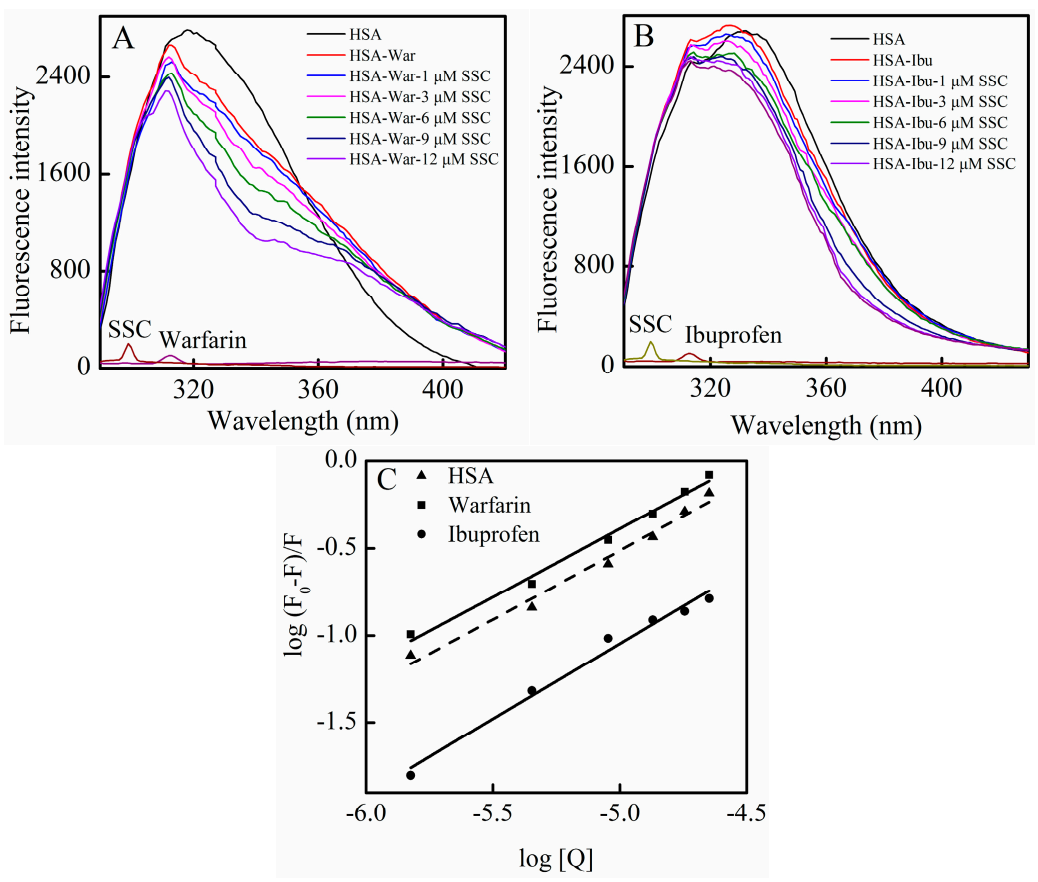

Figure 6. Effect of site marker probes on the SSC-HSA system. The experiment was carried out at $\mathrm{C}_{\mathrm{HSA}}=2 \mu \mathrm{M}, \mathrm{T}=26^{\circ} \mathrm{C}, \mathrm{pH}=7.4$, and $\lambda_{\mathrm{ex}}=280 \mathrm{~nm}$. (A) $\mathrm{C}_{\text {warfarin }}=0.025 \mathrm{M}$; (B) $\mathrm{C}_{\text {ibuprofen }}=0.25 \mathrm{M}$; (C) Double-reciprocal plots for $\log \left(\mathrm{F}_{0}-\mathrm{F}\right) / \mathrm{F}$ vs. $\log [\mathrm{Q}]$; [Q], $\mathrm{C}_{\mathrm{SSC}}=0,1,3,6$, 9, and $12 \mu \mathrm{M}$. War, Warfarin; Ibu, Ibuprofen.

According to the Equation (4), the corresponding plot can be obtained from the fluorescence data (Figure 6C) [28]. The $K_{a}$ in the warfarin or ibuprofen mixture was $4.38 \times 10^{4} \mathrm{~L}^{\circ} \mathrm{mol}^{-1}$ or $^{-3}$ $2.22 \times 10^{3} \mathrm{~L} \cdot \mathrm{mol}^{-1}$, respectively (Table 3 ). Although the binding constants in the study system are low, lower binding constants (about $10^{3} \mathrm{~L} \cdot \mathrm{mol}^{-1}$ ) have also been reported for protein-ligand interactions [29]. Commonly, weak binding to the protein results in a shorter lifetime or poor distribution of drugs in plasma, whereas the strong binding reduces the levels of free plasma drugs [10]. The results showed that the $K_{a}$ of the warfarin complex was much higher than that of SSC-HSA binding, whereas the $K_{a}$ of the ibuprofen complex is below the binding constant of SSC and HSA (Table 3). Confusedly, the $K_{a}$ in the mixture system is higher in the presence of warfarin. Obviously, the effect requires further elucidation in the future. Albumin-binding events play an essential role in the transportation, distribution and free fraction of drugs. Free drugs are responsible for pharmacological activities [30]. The calculated results show that the effect of warfarin or ibuprofen is of practical significance in clinical application. If warfarin is given at the same time with SSC, the warfarin-HSA binding will be able to regulate the binding of SSC and HSA, extend the retention time in plasma, decrease free concentration, and inhibit the effect of SSC/Chaihu. If ibuprofen combines with SSC at the same time, the ibuprofen-HSA interaction can influence SSC and HSA binding, elevate the free concentration, and enhance the effect of SSC/Chaihu. Warfarin, a coumarin representative, and other coumarin anticoagulants, such as acenocoumarol and phenprocoumon, widely exist, not only among Chinese herbal medicines, but also among prescribed drugs worldwide [31]. Ibuprofen, a classical anti-inflammatory drug, and its derivatives are also among the most common prescribed drugs in the world [32]. Chaihu enriching SSC is often prescribed as a major herbal medicine both in some classical 
Chinese medicinal formulae, such as "xiao yao powder", and in any prescription, such as Chaihu and Scutellaria Baicalensis, Chaihu and peony, and Chaihu and Liquorice [33]. Inevitably, with the extensive application of Chaihu in clinical practice, SSC commonly interacts not only with co-administered medicinal herbs, but also likely with co-treated modern drugs. In short, the effect of SSC/Chaihu is likely weakened by warfarin-class drugs, whereas the effect of SSC/Chaihu is likely strengthened when ibuprofen-class drugs and SSC/Chaihu are co-administered.

Table 3. Binding reactive parameters in site competitive experiments.

\begin{tabular}{cccc}
\hline System & Equation & $\boldsymbol{K}_{a}\left(\mathbf{L} \cdot \mathbf{~ m o l}^{-1}\right) \pm \mathbf{S D}$ & $\mathbf{R}$ \\
\hline Warfarin & $\mathrm{Y}=0.78 \mathrm{X}+3.64$ & $4.38 \times 10^{4} \pm 11.65^{*}$ & 0.991 \\
Ibuprofen & $\mathrm{Y}=0.87 \mathrm{X}+3.35$ & $2.22 \times 10^{3} \pm 3.75^{*}$ & 0.985 \\
HSA-SSC & $\mathrm{Y}=0.79 \mathrm{X}+3.57$ & $3.72 \times 10^{3} \pm 4.76$ & 0.983 \\
\hline
\end{tabular}

$K_{a}$, binding constant; $\mathrm{R}$, linear correlation coefficient; SD, standard deviation; * $p<0.05$ compared with HSA-SSC control by the paired $t$-test.

\subsubsection{Binding Forces between SSC and HSA}

The binding forces between a small molecule and a macromolecule can include several types of interactions, such as hydrogen bonds, van der Waals forces, electrostatic forces, and hydrophobic interactions [34,35]. The thermodynamic parameters, enthalpy change $(\Delta H)$ and entropy change $(\Delta S)$, are the major indicators to confirm binding modes. Based on a number of experimental results, Ross summarized the thermodynamic relationship between proteins and small molecules as follows: $\Delta H<0$ and $\Delta S<0$ indicates van der Waals force or hydrogen bond formation; $\Delta H<0$ and $\Delta S>0$ implies an electrostatic force; and $\Delta H>0$ and $\Delta S>0$ reflects hydrophobic interaction [36].

The enthalpy change can be considered as a constant when the effect of tested temperatures on binding constants is not statistically significant (Table 2). From the temperature dependence of the binding constants, the parameters involved in the process were calculated using the following equations (Table 4) [37-39]:

$$
\begin{aligned}
\ln \frac{K_{a 2}}{K_{a 1}} & =\frac{\Delta H}{R}\left(\frac{1}{T_{1}}-\frac{1}{T_{2}}\right) \\
\Delta G & =-R T \ln K_{a} \\
\Delta G & =\Delta H-\Delta T S
\end{aligned}
$$

where $K_{a}$ is the binding constant calculated by Equation (4), and $R$ is the gas constant. As shown in Table 4 , the negative value of the free energy change $(\Delta G)$ means a spontaneous binding process. Both negative enthalpy and entropy values reveal that van der Waals interactions and hydrogen bonds play a major role in the binding process between SSC and HSA (Table 4). Therefore, the interaction between SSC and HSA is a forward reaction involving the reduction of free energy change, and enthalpy change and entropy change at a low temperature, which is mainly driven by enthalpy because the entropy value was negative and unfavorable for the binding process.

Table 4. Thermodynamic parameters of SSC-HSA interaction.

\begin{tabular}{cccccc}
\hline Fluorescence & $\mathbf{T}\left({ }^{\circ} \mathbf{C}\right)$ & $\lambda_{\text {ex }}$ & $\Delta G\left(\mathbf{k J} \cdot \mathbf{~ m o l}^{-\mathbf{1}}\right)$ & $\boldsymbol{\Delta H}\left(\mathbf{k J} \cdot \mathbf{~ m o l}^{-\mathbf{1}}\right)$ & $\Delta \boldsymbol{S}\left(\mathbf{J} \cdot \mathbf{m o l}^{-\mathbf{1}} \cdot \mathbf{K}^{-\mathbf{1}}\right)$ \\
\hline Conventional & 26 & $280 \mathrm{~nm}$ & -20.34 & -47.34 & -90.31 \\
& 36 & $280 \mathrm{~nm}$ & -19.52 & -90.04 \\
\hline
\end{tabular}

\subsection{Energy Transfer between HSA and SSC}

Energy transfer, one of the most conspicuous intermolecular events of the interaction between drugs and albumin, is an electrodynamic process in which a primary excited-state donor can transfer 
energy to its neighbors through a non-radiative dipole-dipole coupling [38]. The process takes place under the following conditions. First, the donor can produce fluorescent light. Second, there is a fair amount of overlap between the fluorescence emission spectra of the donor and the UV-vis absorbance spectra of the acceptor. Additionally, the distance between the donor and the acceptor approaches less than $8 \mathrm{~nm}$ if fluorescence emitted from a donor can be absorbed by an acceptor [40,41]. The phenomenon of energy transfer is associated not only with the distance between the donor and the acceptor, but also with the critical energy transfer distance. According to the Förster mechanism of non-radiative energy transfer, the energy transfer efficiency, and the distance between the donor (HSA) and the acceptor (SSC) can be calculated by the following equation [38]:

$$
E=1-\frac{F}{F_{0}}=\frac{R_{0}^{6}}{R_{0}^{6}+r^{6}}
$$

where $E$ represents the efficiency of transfer between the donor and the acceptor, which can be measured experimentally by the fluorescence emission from donors with $(F)$ and without the acceptor $\left(F_{0}\right)$, and normalized to the same donor concentration [17]. Here, $r$ is the average distance between donor and acceptor. $R_{0}$ is the critical distance when the efficiency of transfer is $50 \%$, which relates to the quantum yield of the donor, the quenching coefficient of the acceptor, the overlap between the fluorescence emission from the donor and absorption spectra from the acceptor, and the orientation of the fluorophore. The calculation for $R_{0}$ is described in the following expression [42]:

$$
R_{0}^{6}=8.79 \times 10^{-25} K^{2} n^{-4} J \varphi
$$

where $K^{2}$, the spatial orientation factor between the emission dipole of the donor and the absorption dipole of the acceptor, is the least certain parameter in the calculation of the critical transfer distance. If both the donor and the acceptor rotate rapidly without any orientation in a supposition, then $K^{2}=2 / 3$. If only the donor is free to spin, then $K^{2}$ ranges from $1 / 3$ to $4 / 3$ [43]. Here, $n$ is the average refractive index of medium in the wavelength range where spectral overlap is significant, and $\varphi$ is the fluorescence quantum yield of the donor. In the present work, $K^{2}=2 / 3, n=1.336, \varphi=0.118$ [44]. J is the overlap integral of the fluorescence emission spectrum of the donor and the absorption spectrum of the acceptor, given by the equation below [22]:

$$
J=\frac{\sum F(\lambda) \varepsilon(\lambda) \lambda^{4} \Delta \lambda}{\sum F(\lambda) \Delta \lambda}
$$

In Equation (10), $F(\lambda)$ is the fluorescence intensity of the fluorescent donor at wavelength $\lambda$, and is dimensionless. Here, $\varepsilon(\lambda)$ is the molar absorption coefficient of the acceptor at wavelength $\lambda$. The overlap integral $(J)$ could be evaluated by integrating the spectra in Figure 7. Based on these data, $J, R_{0}, E$ and $r$ could be obtained between the donor and the acceptor (Table 5). The average distances from a donor to an acceptor in HSA, $\Delta \lambda=15 \mathrm{~nm}$, and $\Delta \lambda=60 \mathrm{~nm}$ are all on the 2 to $8 \mathrm{~nm}$ scale, and $0.5 R_{0}<r<1.5 R_{0}[45]$, which indicates that non-radiative energy transfer occurs with high probability between HSA and SSC, and re-confirms that a static quenching interaction arises between SSC and HSA $[36,46]$.

Table 5. Energy transfer parameters between SSC and HSA.

\begin{tabular}{lcccccc}
\hline Fluorescence & $\mathbf{T}\left({ }^{\circ} \mathbf{C}\right)$ & Detection & $\boldsymbol{J}\left(\mathbf{c m}^{\mathbf{3}} \cdot \mathbf{L} \cdot \mathbf{~ m o l}^{-1}\right)$ & $\boldsymbol{R}_{\mathbf{0}}(\mathbf{n m})$ & $\boldsymbol{E}(\mathbf{J})$ & $\boldsymbol{r}(\mathbf{n m})$ \\
\hline Conventional & 26 & $\lambda_{\text {ex }}=280 \mathrm{~nm}$ & $6.54 \times 10^{-16}$ & 2.14 & 0.23 & 2.61 \\
& 36 & $\lambda_{\mathrm{ex}}=280 \mathrm{~nm}$ & $3.06 \times 10^{-16}$ & 1.89 & 0.096 & 2.74 \\
\multirow{3}{*}{ Synchronous } & 26 & $\Delta \lambda=15 \mathrm{~nm}$ & $1.14 \times 10^{-15}$ & 2.34 & 0.037 & 4.03 \\
& 26 & $\Delta \lambda=60 \mathrm{~nm}$ & $2.65 \times 10^{-15}$ & 2.69 & 0.19 & 3.45 \\
\hline
\end{tabular}



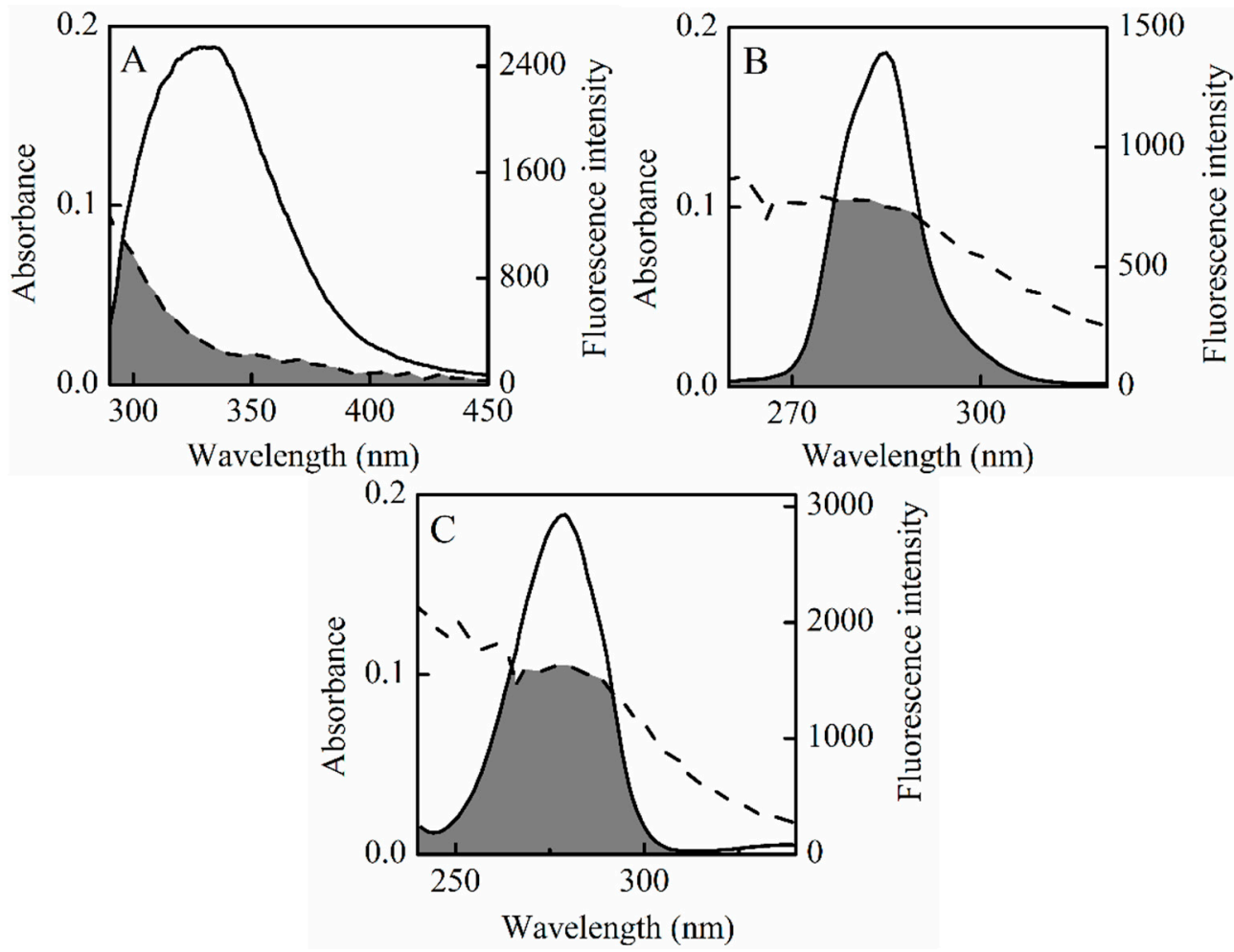

Figure 7. Overlap of the UV absorption spectra of SSC and the fluorescence emission spectra. Dotted line is the UV absorption spectra of $3 \mathrm{mM} \mathrm{SSC}$. (A-C) is the fluorescence emission spectra of $\mathrm{C}_{\mathrm{HSA}}=2 \mu \mathrm{M}, \Delta \lambda=15 \mathrm{~nm}$ or $\Delta \lambda=60 \mathrm{~nm}$, respectively.

\subsection{Docking Results}

Figure 8 shows the theoretical binding mode of SSC to HSA. Trp214, Tyr150 and Tyr411 were located surrounding the hydrophobic pockets in Figure 8B,C, and tyrosine or tryptophan residues were not found at the interface between sub-domain IIA-IIB in Figure 8D, which corresponds to fluorescence spectra in the study. As shown in Figure 8B, SSC adopted a hairpin conformation to binding inside the pocket of the Sudlow I binding site in subdomain IIA (site I). Only three hydrogen bonds were observed between SSC and Sudlow I. Figure 8C demonstrates that SSC docked into the subdomain IIIA (site II, Sudlow II), and five hydrogen bonds occurred between SSC and Sudlow II. Based on the information provided by the PBD databank, SSC was also docked to the IIA-IIB binding site of HSA (Figure 8D). The glucoside group of SSC fit at the bottom of the HSA pocket and a high density of van der Waals contacts, whereas the bridged-ring group of SSC was positioned within the interface between sub-domain IIA-IIB, and made only a few contacts. Moreover, only three hydrogen bond interactions were observed between SSC and HSA.

The above analysis fits extremely well with the site experimental results and binding forces. Detailed analysis was shown in Table 6. To a greater degree, hydrogen bonds increase the stability of SSC-HSA complexes. Accordingly, it is inferred that the SSC-Sudlow II complex is likely the most stable among the three binding modes. Consistent with the site experimental findings, the present docking studies indicate that SSC binds to site I and II. Moreover, the study found binding of SSC to the interface between subdomain IIA-IIB. These results indicate that docking analysis is helpful for providing a structural basis for the interaction between HSA and SSC. 
A

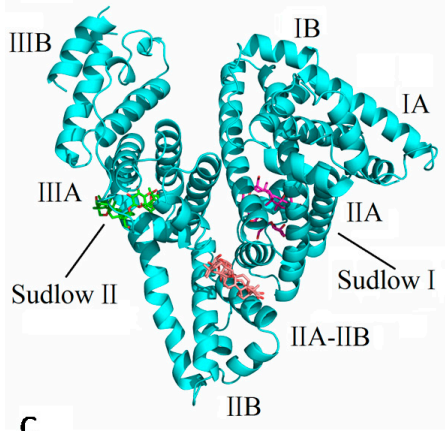

C

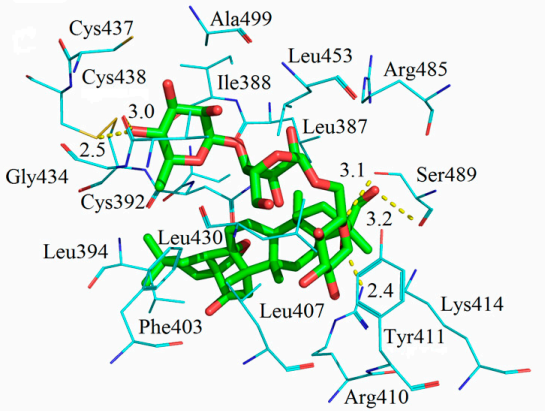

B

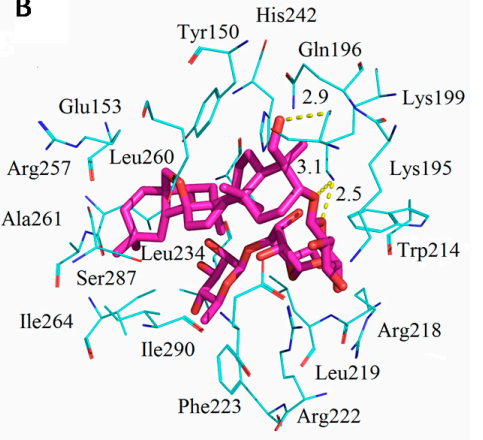

D

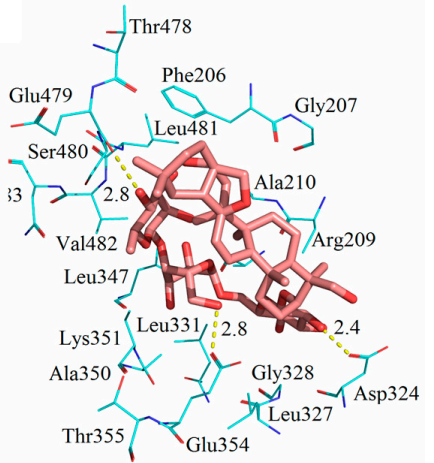

Figure 8. Optimal binding conformation of the SSC-HSA complex (A). Amino acid residues of Sudlow I (B); or Sudlow II (C) or IIA-IIB binding site (D) surrounding SSC within $4 \AA$. The cyan structure stands for HSA. The pink, green, or brown is for SSC, and a dotted line for hydrogen bonding.

Table 6. Atoms and amino acid residues involved in the hydrogen bonds between SSC and HSA.

\begin{tabular}{cccc}
\hline Subdomain & Amino Acid Residues/HSA & Atoms/SSC & Length \\
\hline Sudlow I & Ser192 & $\mathrm{CH}_{2} \mathrm{OH}(19)$ & $2.9 \AA$ \\
& Lys199 & $\mathrm{O}(23)$ & $3.1 \AA$ \\
\multirow{3}{*}{ Sudlow II } & Lys199 & $\mathrm{O}(25)$ & $2.5 \AA$ \\
& Arg-410 & $\mathrm{O}(23)$ & $2.4 \AA$ \\
& Ser489 & $\mathrm{CH}_{2} \mathrm{OH}(19)$ & $3.1 \AA$ \\
& Ser489 & $\mathrm{O}(25)$ & $3.2 \AA$ \\
& Cys438 & $\mathrm{OH}(45)$ & $2.5 \AA$ \\
IIA-IIB & Val433 & $\mathrm{OH}(45)$ & $3.0 \AA$ \\
& Asp324 & $\mathrm{OH}(26)$ & $2.4 \AA$ \\
& Glu354 & $\mathrm{CH}_{2} \mathrm{OH}(37)$ & $2.8 \AA$ \\
& Glu479 & $\mathrm{OH}(45)$ & $2.8 \AA$ \\
\hline
\end{tabular}

*, see Figure 1.

\section{Materials and Methods}

\subsection{Materials}

HSA (approx. 99.0\% purity by electrophoresis), tyrosine and tryptophan (both $98.0 \%$ purity by TLC) were purchased from Sigma (St Louis, MO, USA). SSC (98.0\% purity by HPLC) was obtained from Biopurify Phytochemical Ltd. (Chengdu, Sichuan, China). Ibuprofen and warfarin (both 99.7\% purity) were supplied by the National Institute for Control of Pharmaceutical and Bioproducts (Beijing, China). All other reagents were of analytical grade, and used without further purification. Sample masses were accurately weighed on a microbalance (Sartorius, Gottingen, Germany) with a resolution of $0.01 \mathrm{mg}$. All pH measurements were made with a pH meter (Sartorius, Gottingen, Germany). 


\subsection{Absorbance Measurements}

The UV-vis absorption spectrum was recorded at room temperature on a NanoDrop 2000c spectrophotometer (Thermo, Waltham, MA, USA) equipped with $1.0 \mathrm{~cm}$ quartz cells. Spectrum data points were collected from 280 to $450 \mathrm{~nm}$.

\subsection{Fluorescence Measurements}

\subsubsection{Conventional Fluorescence Measurements for SSC-HSA Binding}

All fluorescence measurements were recorded on a F-7000 spectrofluorimeter (Hitachi, Tokyo, Japan) equipped with $1.0 \mathrm{~cm}$ quartz cells. The widths of the fluorescence excitation slit and emission slit were fixed at $2.5 \mathrm{~nm}$ for all experiments. Scan speed was set at $1200 \mathrm{~nm} \cdot \mathrm{min}^{-1}$. The excitation wavelength was set at $280 \mathrm{~nm}$ [47]. In each assay, $3.0 \mathrm{~mL} \mathrm{HSA}$ at a concentration of $2 \mu \mathrm{M}$ was added into the quartz cell. Subsequently, the HSA solution was titrated by successive additions of a $3 \mathrm{mM}$ stock solution of SSC, using a microsyringe, to a final SSC concentration of $0,1,3,6,9,12$ and $15 \mu \mathrm{M}$. Fluorescence intensities were measured at $26{ }^{\circ} \mathrm{C}(=299 \mathrm{~K})$ and $36{ }^{\circ} \mathrm{C}(=309 \mathrm{~K})$. All samples were prepared in $0.01 \mathrm{M}$ phosphate buffer saline (PBS) at $\mathrm{pH}$ 7.4. Each experiment was repeated three times. Origin 8 SR2 Software (OriginLab, Nothampton, MA, USA) was used for plotting and calculation.

\subsubsection{Synchronous Fluorescence Measurements for SSC-HSA Binding}

Synchronous fluorescence spectroscopy involves the simultaneous scanning of excitation and emission monochromators at a fixed wavelength difference $\left(\Delta \lambda=\lambda_{\mathrm{em}}-\lambda_{\mathrm{ex}}\right)$, with good selectivity and sensitivity, and less interference. The excitation and emission slit widths were fixed at $2.5 \mathrm{~nm}$. The scan speed was set at $240 \mathrm{~nm} \cdot \mathrm{min}^{-1}$. In each assay, $3.0 \mathrm{~mL}$ HSA at $2 \mu \mathrm{M}$ was titrated by successive additions of $3 \mathrm{mM} \mathrm{SSC}$ (concentration from 0 to $15 \mu \mathrm{M}$ ). Synchronous fluorescence was always performed at $26^{\circ} \mathrm{C}$. The spectrum data points were collected with $\Delta \lambda=15 \mathrm{~nm}$ for tyrosine or $\Delta \lambda=60 \mathrm{~nm}$ for tryptophan. Each experiment was repeated three times.

\subsubsection{Synchronous Fluorescence Measurements for SSC-Tyrosine or SSC-Tryptophan Binding}

For tyrosine or tryptophan, $3.0 \mathrm{~mL}$ solutions, at $10 \mu \mathrm{M}$ concentration, were titrated by successive additions of $3 \mathrm{mM}$ SSC solution. The other procedures were the same as Section 3.3.2.

\subsubsection{Site Marker Competitive Fluorescence Experiments}

In the presence of two site markers, warfarin and ibuprofen, binding site studies between SSC and HSA were carried out by conventional fluorescence, as described in Section 3.3.1. Before displacement, $2.0 \mathrm{~mL}$ of $2 \mu \mathrm{M}$ HSA was incubated, at $26^{\circ} \mathrm{C}$ for $30 \mathrm{~min}$, with $0.25 \mathrm{M}$ ibuprofen or $0.025 \mathrm{M}$ warfarin in PBS at pH 7.4. Then, SSC solution was titrated into a $1.0 \mathrm{~cm}$ quartz cuvette by successive additions. For warfarin or ibuprofen solution alone, the fluorescence data were collected in similar fashion as the mixture system. Each experiment was repeated three times.

\subsubsection{Correction of the Internal Filter}

To avoid the effect of the internal filter in the SSC-HSA system on the fluorescence data, the following formula was utilized [8]:

$$
F_{c o r}=F_{o b s} \times 10^{\left(A_{e x}+A_{e m}\right) / 2}
$$

where $F_{c o r}$ and $F_{o b s}$ are the fluorescence intensities after and before the correction. $A_{e x}$ and $A_{e m}$ are the absorbance at the excitation and emission, respectively. 


\subsection{Spectroscopy}

CD measurements of HSA with and without SSC were recorded using a MOS 450 automatic spectropolarimeter (BioLogic Science Instruments, Claix, France) equipped with $1.0 \mathrm{~cm}$ quartz cells at $26^{\circ} \mathrm{C}$, over the scan range of $200-350 \mathrm{~nm}$, at a scan rate of $30 \mathrm{~nm} / \mathrm{min}$ with a response time of $4 \mathrm{~s}$. Data were corrected using the signal of the buffer solution.

\subsection{Molecular Docking}

Molecular docking was performed to investigate the binding mode of SSC to HSA by means of Autodock Vina software (version 1.1.2). The three-dimensional (3D) coordinate of the HSA (PDB ID: 2XVU) was downloaded from the Protein Data Bank (http://www.rcsb.org/pdb) [48]. The Auto Dock Tools version 1.5.6 software (http://mgltools.scripps.edu) was employed to generate the docking input files. Ligand structure was prepared for docking by merging non-polar hydrogen atoms and defining rotatable bonds. In order to increase the docking accuracy, the value of exhaustiveness was set to 20. For Vina docking, the default parameters were used as described in the Autodock Vina manual unless otherwise specified. The top ranked pose as judged by the Vina docking score was subject to visually analysis by using PyMOL 1.7 software (http:/ /www.pymol.org/).

\section{Conclusions}

SSC can specifically bind to HSA and quench HSA's intrinsic fluorescence by a static mechanism. The Stern-Volmer constant is inversely correlated with temperature, indicating that the formation of SSC-HSA complexes is predominantly responsible for the quenching. Thermodynamic parameters indicate that the binding process occurs spontaneously, and that hydrogen bonds and Van der Waals forces play a major role. The calculated binding constants and binding mechanism is consistent with the experimental data. Based on the Förster theory, non-radiation energy transfer can occur. Experimental site studies suggest that SSC can bind to site I (subdomain IIA) and site II (subdomain IIIA) of HSA. The results obtained from synchronous fluorescence, and CD spectroscopy demonstrate that the HSA conformation is affected by SSC when the two bind. Molecular docking studies further confirm the experimental findings in conformational change, binding site and binding force induced by SSC. Besides, SSC is also docked to the interface between subdomain IIA and IIB. Notably, SSC action may be obviously weakened by the co-administration of SSC/Chaihu and warfarin-class drugs, whereas SSC action may be promoted when ibuprofen-class drugs and SSC/Chaihu are co-administered at the same time.

Acknowledgments: This work was supported by the grants from the National Natural Science Foundation of China (No. 81573820 and 81072941) and the department of education, Guangdong government under the top-tier university development scheme for research and control of infectious diseases.

Author Contributions: Yi-Cun Chen and Qing-Xia Niu designed the study. Yi-Cun Chen, Hong-Mei Wang and Dan-Yan Ye analyzed the data. Qing-Xia Niu and Hong-Mei Wang wrote the paper. All authors performed the experiments, discussed the results, and approved the manuscript. Yi-Cun Chen and Hong-Mei Wang contributed equally. Guo-Wu Liang mainly joined in fluorescence experiments.

Conflicts of Interest: The authors declare no conflict of interest.

\section{References}

1. Curry, S.; Mandelkow, H.; Brick, P.; Franks, N. Crystal structure of human serum albumin complexed with fatty acid reveals an asymmetric distribution of binding sites. Nat. Struct. Biol. 1998, 5, 827-835. [CrossRef] [PubMed]

2. Ranjbar, S.; Shokoohinia, Y.; Ghobadi, S.; Bijari, N.; Gholamzadeh, S.; Moradi, N.; Ashrafi-Kooshk, M.R.; Aghaei, A.; Khodarahmi, R. Studies of the interaction between isoimperatorin and human serum albumin by multispectroscopic method: identification of possible binding site of the compound using esterase activity of the protein. Sci. World J. 2013, 2013. [CrossRef] [PubMed]

3. Mahammed, A.; Gary, H.B.; Weaver, J.J.; Sorasaenee, K.; Gross, Z. Amphiphilic corroles bind tightly to human serum albumin. Bioconjugate Chem. 2004, 15, 738-746. [CrossRef] [PubMed] 
4. $\quad$ Simard, J.R.; Zunszain, P.A.; Ha, C.E.; Yang, J.S.; Bhagavan, N.V.; Petitpas, I.; Curry, S.; Hamilton, J.A. Locating high-affinity fatty acid-binding sites on albumin by x-ray crystallography and NMR spectroscopy. Proc. Natl. Acad. Sci. USA 2005, 102, 17958-17963. [CrossRef] [PubMed]

5. Xie, M.X.; Long, M.; Liu, Y.; Qin, C.; Wang, Y.D. Characterization of the interaction between human serum albumin and morin. BBA-Gen. Subj. 2006, 1760, 1184-1191. [CrossRef] [PubMed]

6. Sudhamalla, B.; Gokara, M.; Ahalawat, N.; Amooru, D.G.; Subramanyam, R. Molecular dynamics simulation and binding studies of $\beta$-sitosterol with human serum albumin and its biological relevance. J. Phys. Chem. B 2010, 114, 9054-9062. [CrossRef] [PubMed]

7. Eftink, M.R.; Hagaman, K.A. Fluorescence lifetime and anisotropy studies with liver alcohol dehydrogenase and its complexes. Biochemistry 1986, 25, 6631-6637. [CrossRef] [PubMed]

8. Shi, J.; Wang, J.; Zhu, Y.; Chen, J. Characterization of interaction between isoliquiritigenin and bovine serum albumin: Spectroscopic and molecular docking methods. J. Lumin. 2014, 145, 643-650. [CrossRef]

9. Meneghini, C.; Leboffe, L.; Bionducci, M.; Fanali, G.; Meli, M.; Colombo, G.; Fasano, M.; Ascenzi, P.; Mobilio, S. The five-to-six-coordination transition of ferric human serum heme-albumin is allosterically-modulated by ibuprofen and warfarin: A combined XAS and MD study. PLoS ONE 2014, 9, e104231.

10. Samari, F.; Shamsipur, M.; Hemmateenejad, B.; Khayamian, T.; Gharaghani, S. Investigation of the interaction between amodiaquine and human serum albumin by fluorescence spectroscopy and molecular modeling. Eur. J. Med. Chem. 2012, 54, 255-263. [CrossRef] [PubMed]

11. Zhu, Z.B.; Liang, Z.S.; Hu, R.L.; Dong, J.E. Growth and Saikosaponin production of the medicinal herb Bupleurum chinense DC. Under different levels of nitrogen and phosphorus. Ind. Crop. Prod. 2009, 29, 96-101. [CrossRef]

12. Chiang, L.C.; Ng, L.T.; Liu, L.T.; Shieh, D.E.; Lin, C.C. Cytotoxicity and anti-hepatitis B virus activities of Saikosaponins from Bupleurum species. Planta Med. 2003, 69, 705-709. [PubMed]

13. Law, B.Y.; Mo, J.F.; Wong, V.K. Autophagic effects of Chaihu (dried roots of Bupleurum chinense DC or Bupleurum scorzoneraefolium WILD). Chin. Med. 2014, 9. [CrossRef] [PubMed]

14. Que, R.; Li, Y. Advances in mechanisms of Saikosaponin preventing and treating hepatocellular carcinoma. J. Liaoning Univ. TCM 2014, 16, 128-131. (in Chinese).

15. Shyu, K.; Tsai, S.; Wang, B.; Liu, Y.; Lee, C. Saikosaponin C induces endothelial cells growth, migration and capillary tube formation. Life Sci. 2004, 76, 813-826. [CrossRef] [PubMed]

16. Lee, T.H.; Chang, J.; Kim, B.M. Saikosaponin C inhibits lipopolysaccharide-induced apoptosis by suppressing caspase-3 activation and subsequent degradation of focal adhesion kinase in human umbilical vein endothelial cells. Biochem. Biophys. Res. Commun. 2014, 445, 615-621. [CrossRef] [PubMed]

17. Hu, J.Y.; Liu, Y.; Xiao, X.H. Investigation of the interaction between berberine and human serum albumin. Biomacromolecules 2009, 10, 517-521. [CrossRef] [PubMed]

18. Cohen, B.E.; Pralle, A.; Yao, X.; Swaminath, G.; Gandhi, C.S.; Jan, Y.N.; Kobilka, B.K.; Isacoff, E.Y.; Jan, L.Y. A fluorescent probe designed for studying protein conformational change. Proc. Natl. Acad. Sci. USA 2005, 102, 965-970. [CrossRef] [PubMed]

19. Jing, M.; Xie, M.X.; Zheng, D.; Liu, Y.; Li, X.Y.; Chen, X. Spectroscopic studies on the interaction of cinnamic acid and its hydroxyl derivatives with human serum albumin. J. Mol. Struct. 2004, 692, 71-80.

20. Kang, J.; Liu, Y.; Xie, M.X.; Li, S.; Jiang, M.; Wang, Y.D. Interactions of human serum albumin with chlorogenic acid and ferulic acid. BBA-Gen. Subj. 2004, 1674, 205-214. [CrossRef] [PubMed]

21. Zhao, L.; Liu, R.; Zhao, X.; Yang, B.; Gao, C.; Hao, X.; Wu, Y. New strategy for the evaluation of CdTe quantum dot toxicity targeted to bovine serum albumin. Sci. Total Environ. 2009, 407, 5019-5023. [CrossRef] [PubMed]

22. Mahesha, H.G.; Singh, S.A.; Srinivasan, N.; Rao, A.G.A. A spectroscopic study of the interaction of isoflavones with human serum albumin. FEBS J. 2006, 273, 451-467. [CrossRef] [PubMed]

23. Carter, D.C.; Ho, J.X. Structure of serum albumin. Protein Chem. 1994, 45, 153-203.

24. Ji, Z.; Yuan, H.; Liu, M.; Hu, J. 1H-NMR study of the effect of acetonitrile on the interaction of ibuprofen with human serum albumin. J. Pharm. Biomed. Anal. 2002, 30, 151-159. [CrossRef]

25. Sugio, S.; Kashima, A.; Mochizuki, S.; Noda, M.; Kobayashi, K. Crystal structure of human serum albumin at 2.5 A resolution. Protein Eng. 1999, 12, 439-446. [CrossRef] [PubMed]

26. Goncharova, I.; Orlov, S.; Urbanova, M. The location of the high- and low- affinity bilirubin-binding sites on serum albumin: Ligand-competition analysis investigated by circular dichroism. Biophys. Chem. 2013, 180-181, 55-65. [CrossRef] [PubMed] 
27. Li, Y.; He, W.; Liu, J.; Sheng, F.; Hu, Z.; Chen, X. Binding of the bioactive component Jatrorrhizine to human serum albumin. BBA-Gen. Subj. 2005, 1722, 15-21. [CrossRef] [PubMed]

28. Maiti, T.K.; Ghosh, K.S.; Debnath, J.; Dasgupta, S. Binding of all-trans retinoic acid to human serum albumin: Fluorescence, FT-IR and circular dichroism studies. Int. J. Biol. Macromol. 2006, 38, 197-202. [CrossRef] [PubMed]

29. Cheng, T.G.; Li, Q.L.; Zhou, Z.G.; Wang, Y.L.; Bryant, S.H. Structure-Based Virtual Screening for Drug Discovery: A Problem-Centric Review. AAPS J. 2012, 14, 133-141. [CrossRef] [PubMed]

30. Soltes, L.; Mach, M. Estimation of drug-protein binding parameters on assuming the validity of thermodynamic equilibrium. J. Chromatogr. B 2002, 768, 113-119. [CrossRef]

31. Daly, A.K. Optimal dosing of warfarin and other coumarin anticoagulants: The role of genetic polymorphisms. Arch. Toxicol. 2013, 87, 407-420. [CrossRef] [PubMed]

32. Lesko, S.M.; Mitchell, A.A. An assessment of the safety of pediatric ibuprofen. A practitioner-based randomized clinical trial. J. Pediatr. 1995, 273, 836. [CrossRef]

33. Zhang, Z. Exploration of Chaihu couplet medicines in Shang Han Lun. Hubei J. TCM 2014, 36, 47-48. (in Chinese).

34. Tian, J.N.; Liu, J.Q.; He, W.Y.; Hu, Z.D.; Yao, Z.D.; Chen, X.G. Probing the binding of scutellarin to human serum albumin by circular dichroism, fluorescence spectroscopy, FTIR, and molecular modeling method. Biomacromolecules 2004, 5, 1956-1961. [CrossRef] [PubMed]

35. Klotz, I.M.; Urquhart, J.M. Effect of temperatrue on binding of organicions by proteins. J. Am. Chem. Soc. 1949, 71, 847-865. [CrossRef]

36. Ross, P.D.; Subramanian, S. Thermodynamics of protein association reactions: Forces contributing to stability. Biochemistry 1981, 20, 3096-3102. [CrossRef] [PubMed]

37. Zhao, X.C.; Liu, R.T.; Chi, Z.X.; Teng, Y.; Qin, P.F. New Insights into the Behavior of Bovine Serum Albumin Adsorbed onto Carbon Nanotubes: Comprehensive Spectroscopic Studies. J. Phys. Chem. B. 2010, 114, 5625-5631. [CrossRef] [PubMed]

38. Chatterjee, T.; Pal, A.; Dey, S.; Chatterjee, B.K.; Chakrabarti, P. Interaction of virstatin with human serum albumin: Spectroscopic analysis and molecular modeling. PLoS ONE 2012, 7, e37468. [CrossRef] [PubMed]

39. Chen, J.; Zhou, X.; Zhang, Y.; Qian, Y.; Gao, H. Interactions of acidic pharmaceuticals with human serum albumin: Insights into the molecular toxicity of emerging pollutants. Amino Acids 2012, 43, 1419-1429. [CrossRef] [PubMed]

40. Jiang, X.Y.; Li, W.X.; Cao, H. Study of the interaction between trans-resveratrol and BSA by the multispectroscopic method. J. Solut. Chem. 2008, 37, 1609-1623. [CrossRef]

41. Xiao, J.B.; Chen, X.Q.; Jiang, X.Y.; Hilczer, M.; Tachiya, M. Probing the interaction of trans-resveratrol with bovine serum albumin: A fluorescence quenching study with tachiya model. J. Fluoresc. 2008, 18, 671-678. [CrossRef] [PubMed]

42. Shahabadi, N.; Maghsudi, M.; Rouhani, S. Study on the interaction of food colourant quinoline yellow with bovine serum albumin by spectroscopic techniques. Food Chem. 2012, 135, 1836-1841. [CrossRef] [PubMed]

43. Wu, C.W.; Stryer, L. Proximity relationships in rhodopsin. Proc. Natl. Acad. Sci. USA. 1972, 69, $1104-1108$. [CrossRef] [PubMed]

44. Xiao, J.B.; Shi, J.; Cao, H.; Wu, S.D.; Ren, F.L.; Xu, M. Analysis of binding interaction between puerarin and bovine serum albumin by multi-spectroscopic method. J. Pharmaceut. Biomed. 2007, 45, 609-615. [CrossRef] [PubMed]

45. Lakowicz, J.R. Principles of fluorescence spectroscopy. Springer 2006, 15, 518-520.

46. Weiss, S. Fluorescence spectroscopy of single biomolecules. Science 1999, 283, 1676-1683. [CrossRef] [PubMed]

47. Möller, M.; Denicola, A. Protein tryptophan accessibility studied by fluorescence quenching. Biochem. Mol. Biol. Educ. 2002, 30, 175-178. [CrossRef]

48. RCSB Protein Data Bank-Structure Summary. Available online: http://www.rcsb.org/pdb/explore.do? structureId=2XVU (accessed on 30 October 2015).

Sample Availability: Samples of saikosaponin C from Bupleurum chinense DC are available from the authors.

(C) 2016 by the authors; licensee MDPI, Basel, Switzerland. This article is an open access article distributed under the terms and conditions of the Creative Commons by Attribution (CC-BY) license (http://creativecommons.org/licenses/by/4.0/). 